\title{
GIS-supported evaluation of landslide susceptibility in the karst mountainous area: A case study in Wudang, Guiyang
}

\author{
Song $\mathrm{Li}^{1,2^{*}}$, Yi $\mathrm{Bai}^{1}$, Yongjun Long ${ }^{1}$ and Maoqiang $\mathrm{Wang}^{3}$ \\ ${ }^{1}$ Guizhou Provincial Key Laboratory of Watershed Geographical Conditions, Guiyang 550018, China \\ ${ }^{2}$ Institute of environment, resources and disaster Guizhou Education College, Guiyang Guizhou 550018, China \\ ${ }^{3}$ School of Geographical and Environmental Sciences, Guizhou Normal University, Guiyang 550001, China
}

\begin{abstract}
Landslide is the main disaster in the mountainous area. Based on landslide information content models of remote sensing, the work used the aerial and space remote sensing of UltraCamXp WA, Beijing-1 and Landsat images in Wudang, Guiyang to obtain the relative relief, slope, curvature of bedding slope, LUCC, geology and TWI. Finally, we analyzed the spatial susceptibility in the research area. Results showed that there were 42, 56 and 46 potential landslide groups in the high, higher and medium risk regions. The controlling factors of landslides in Wudang, Guiyang refer to the precipitation and precipitation intensity. The densely-populated regions also have the high risk of landslide, and the risk of landslide generally decreases from cities to rural areas. Through the space prediction research of landslide disasters, it is expected to provide valuable protection for regional security and harmonious development, and then sustainable development of Guizhou Province.
\end{abstract}

\section{Introduction}

As the main type of disasters in mountain areas, landslide can be predicted in the space and time dimensions. There are qualitative and quantitative methods for landslide space prediction. Depending on expert experiences, the qualitative and semi-qualitative methods have strong subjective severance ${ }^{[1-2]}$. In contrast, the quantitative method shows the advantages of objectivity and efficiency by exploring the quantitative factors in landslides. Probability and statistical methods are commonly used in the landslide space prediction ${ }^{[3-5]}$. According to comparative analysis of three methods, ANN has better application effect than logistic regression and frequency ratio methods ${ }^{[5-9]}$. Landslide prediction is greatly affected by the selection of landslide influencing factors, which varies depending on the region, type and researcher ${ }^{[9-11]}$. General landslide influencing factors consist of precipitation, slope, aspect, land type, lithology and structure. Besides, the precipitation characteristics affect the landslide spatial prediction ${ }^{[12-14]}$. Tongzhen $\operatorname{Yan}^{[15]}$ divided the landslide prediction theory into deterministic, statistical analysis and information models, where the first two essentially belong to the white and black box models; the rest is somewhere between white and black boxes. The information content model was used to analyze the landslide space prediction in Wudang(fig.1), Guiyang with urban and rural characteristics in Karst mountainous areas. Through spatial prediction research of landslide disasters, it is expected to provide valuable protection for regional security and harmonious development.

\section{Materials and Methods}

\subsection{Landslide factors}

This study area is located at Northeast of Guiyang, and coordinates of latitude and longitud are $106.718^{\circ} \mathrm{E}-$ $107.049^{\circ} \mathrm{E}, 26.570^{\circ} \mathrm{N}-26.911^{\circ} \mathrm{N}$, respectively.The area of this study area is $686 \mathrm{~km}^{2}$. The factor analysis method is used to determine the landslide factors and find the main factors. The $m$ variables are expressed as the linear combinations of factors as follows.

$$
X=A F+C U
$$

where $X$ is the original variable represented by Matrix $p \times 1 ; F$ the common factor by Matrix $k \times 1 ; A$ the factor load matrix by Matrix $p \times k$; $U$ the special of each variable by Matrix $p \times 1 ; C=\left[\begin{array}{ccc}\mathrm{c}_{1} & \cdots & \cdots \\ \cdots & \mathrm{c}_{2} & \cdots \\ \cdots & \cdots & \mathrm{c}_{3}\end{array}\right]$ the single factor load matrix. In principal component analysis, the principal factor model is summarized as $X=A F$ (the meaning is the same as above). Through the principal component analysis, few factors containing most information are found to analyze the essence of landslide. The factor load can be calculated by Equation (2).

$$
a_{i j}=a_{i j} \sqrt{\lambda_{j}}
$$

\footnotetext{
*Song Li: zhijinese@163.com
} 
$a_{i j}$ is the feature vector $p(j=1,2, \ldots, m)$ corresponding to feature value $\lambda(j=1,2, \ldots m)$, expressed as a unit vector; $\sqrt{\lambda_{j}}$ is the square root of the $j^{\text {th }}$ feature value, indicating the importance of factor.

\subsection{Landslide information model}

The information content model is used to analyze the landslide space prediction in research area (Equation (3)).

$$
\mathrm{I}\left(\mathrm{y}, x_{1}, x_{2}, \ldots, x_{n}\right)=\log _{2} \frac{\mathrm{P}\left(\mathrm{y}, x_{1}, x_{2}, \ldots, x_{n}\right)}{\mathrm{P}(\mathrm{y})}
$$

As the terms in statistical probability, $\mathrm{P}\left(\mathrm{y}, x_{1}, x_{2}, \ldots, x_{\mathrm{n}}\right)$ and $\mathrm{P}(\mathrm{y})$ represent the information content by the landslide factors $x_{1}, x_{2}, \ldots, x_{\mathrm{n}}$. When $\mathrm{P}\left(\mathrm{y}, x_{1}, x_{2}, \ldots, x_{\mathrm{n}}\right)>\mathrm{P}(\mathrm{y})$, then the information content $\mathrm{I}\left(\mathrm{y}, x_{1}, x_{2}, \ldots, x_{\mathrm{n}}\right)>0$, indicating the high probability of landslide occurrence. When $\mathrm{I}\left(\mathrm{y}, x_{1}, x_{2}, \ldots, x_{\mathrm{n}}\right)<0$, there is low probability of landslide occurrence. Geographical cells are classified according to geology, topography, hydrology and LUCC. After dividing the research area into $N$ model units, there are $N_{o}$ units with landslide. There are $\mathrm{M}_{0}$ units having landslide disasters among $M$ combined units of $x_{1}, x_{2}, \ldots$, $x_{\mathrm{n}}$ with the same attribute.

In general, landslide is the comprehensive effect of regional multi-factors. Considering the multi-factors, Equation (4) can be transformed into Equation (5).

$$
\begin{gathered}
\mathrm{I}\left(\mathrm{y}, x_{1}, x_{2}, \cdots, x_{n}\right)=\sum_{i=1}^{n} \log _{2} \frac{M_{0} / M}{N_{0} / N} \\
\mathrm{I}=\sum_{i=1}^{n} \mathrm{I}_{i}=\sum_{i=1}^{n} \log _{2} \frac{M_{0}^{i} / M^{i}}{N_{i} / N}
\end{gathered}
$$

After screening, 6 key landslide factors are determined, including relative height difference, slope, forward slope

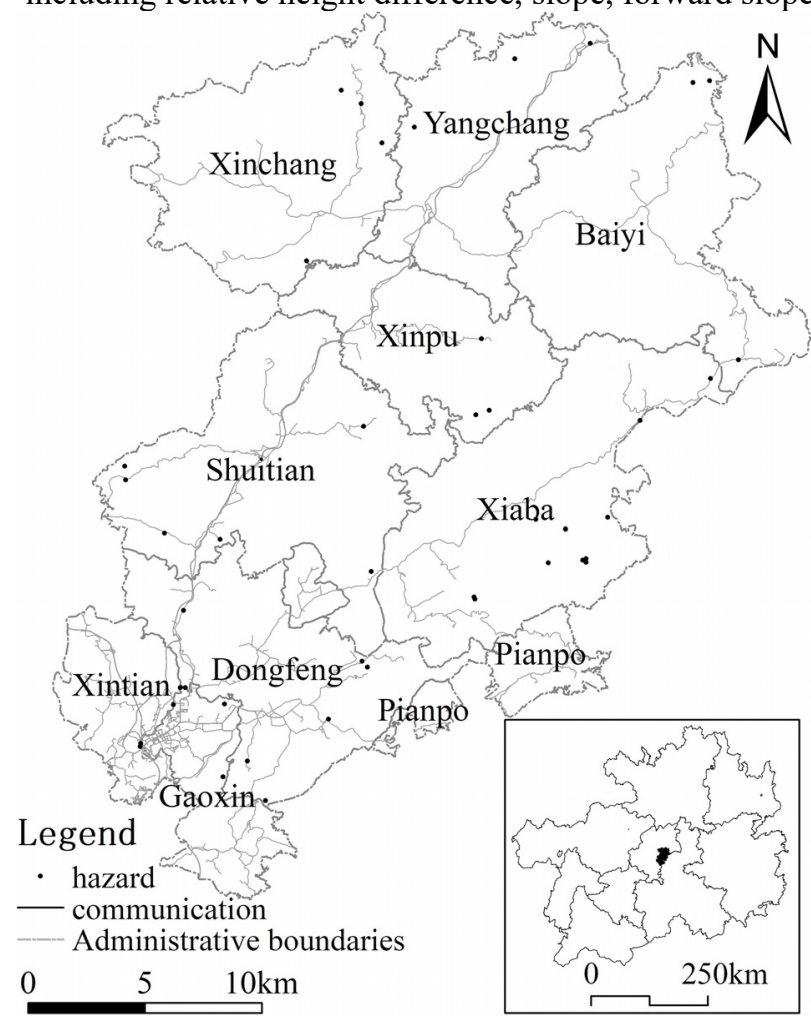

Fig.1. Study area and its location map curvature, land use/cover change (LUCC), geological and topographical wetness index (TWI).

\section{Results and analysis}

\subsection{Landslide factors}

After vectorizing the 1:200000 geological map, the $30 \mathrm{~m} \times 30 \mathrm{~m}$ lattice was used for meshing according to the 1:50000 topographic map and digital elevation model (DEM, 30m). Before the information extraction and subsequent processing, other basic data were conducted with relative geometric correction based on the topographic map. After that, the DEM was used to extract relative height difference, slope, forward slope curvature and TWI. Landsat 8 OLI was used for the extraction of LUCC.

\subsection{Results and analysis}

Based on the spatial analysis function of ArcGIS, the single factor information content of each landslide factor was calculated by Equation (4). Only the information content calculation and classification parts were listed for the LUCC and geological factors. Then, the single factor information content calculated by Equation (4) was synthesized to obtain the comprehensive landslide information content in research area. After calculation, the landslide information content was 0-10.76 in research area. Combined with the Nature Break method and the group-occurring landslides, the landslide information content was divided into higher risk region $(4.00,10.76]$, high risk region $(2.50,4.00]$, medium risk region $(1.50,2.50]$, lower risk region $(0.70,1.50]$ and low risk region $(0,0.70]$. Wherein, the high risk region had a total area of $4.92 \mathrm{~km}^{2}$, with 42 potential landslide groups. The higher risk region had 56 potential landslide groups, covering a total area of $52.25 \mathrm{~km}^{2}$. The middle risk region had a total area of $165.16 \mathrm{~km}^{2}$, with 46 landslide groups. The lower and low risk regions had the areas of 202.66 and $261.01 \mathrm{~km}^{2}$, respectively. The total area was $686.00 \mathrm{~km}^{2}$ (fig.2).

For LUCC, the medium-coverage, low-coverage grassland and sparse forest land had the higher landslide risk and large landslide density, with the information contents of $0.932,1.621$ and 1.240 , respectively. Oppositely, the forest land was the most stable region. Therefore, the vegetation was important to the stability of landslides. The region with higher TWI value had greater development potential of saturation region. It easily reached saturation and formed a weak region, leading to the high risk of landslide. In Wudang, the region of TWI $>7$ had the highest risk of landslide, with the landslide information content of 0.200 . The region with the information content between 6.0 and 7.0 had the higher risk of landslide, with the landslide information content of 0.167 . The concave and composite slopes had the high risk of landslide, with the information contents of 0.474 and 0.167 , respectively. The relative height 


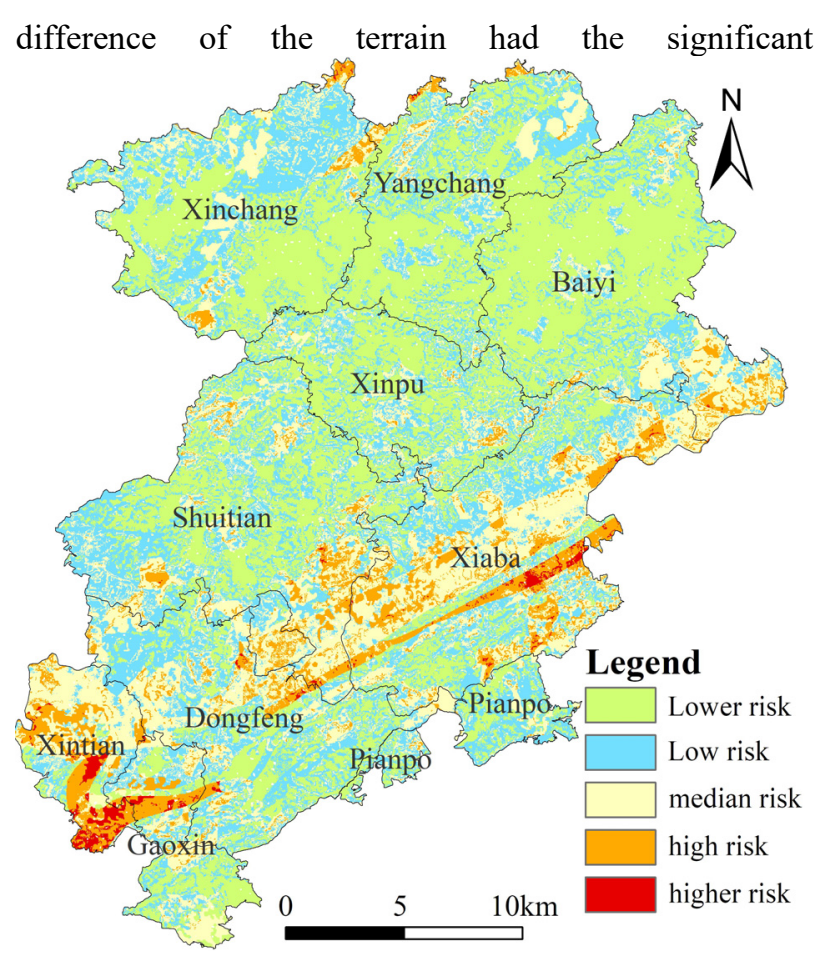

Fig.2. Landslide susceptibility mapping in Wudang, Guiyang

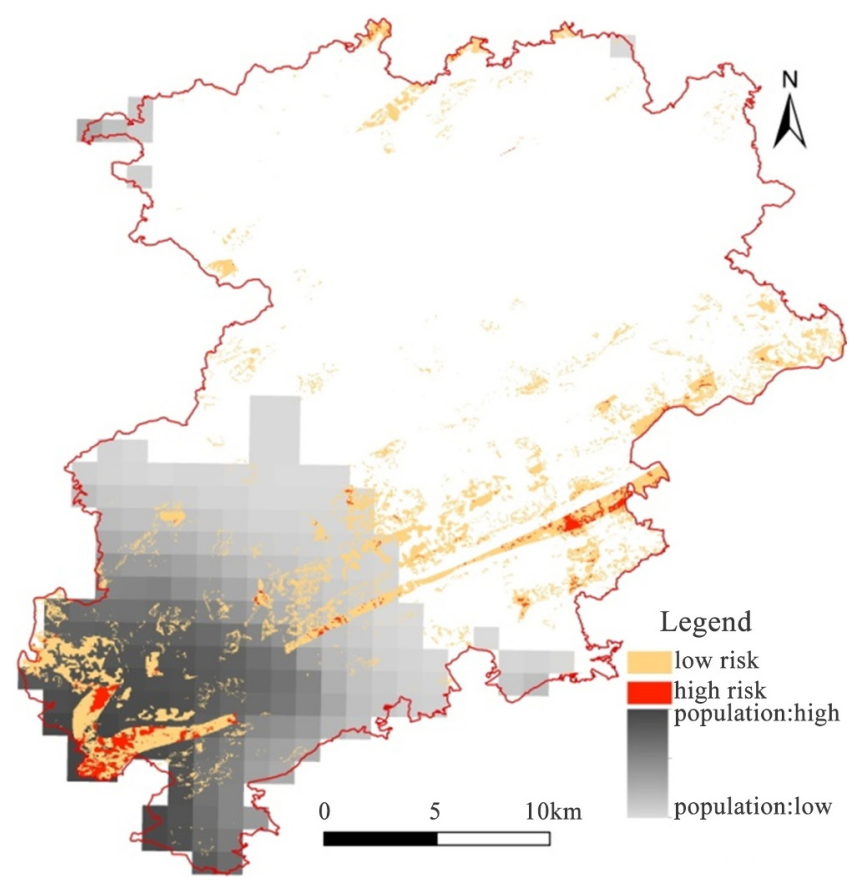

Fig.3. Overlaying analysis of landslide risk and night-time light

correlation with the risk of landslide. In the regions with relative height differences of 150-200, 200-250 and 250$300 \mathrm{~m}$, the landslide information contents were 0.209 , 0.363 and 1.168 , respectively. The information content was only -0.806 in the region with relative height difference of less than $100 \mathrm{~m}$. The landslide risk was directly related with the relative height difference. The larger height difference meant the greater landslide information and higher landslide risk. After the field verification of landslide prediction by GPS and SLR cameras, we analyzed the impact of slope landslide risk on regional human activities based on slope stability. The project took the schools with the highest population density and quantity as the analytic targets. Superimposed landslide prediction, school location and 3D terrain (2.5 times magnification) showed that Wudang Shunhai Primary School (106.626N, 26.625E), Guiyang Xintian Driving Training School (106.743N, 26.621E), Quanlin Enterprise Group Automobile Driver Training School (106.747N, 26.622E), Jinjiang Hualei Kindergarten $(106.762 \mathrm{~N}, 26.643 \mathrm{E})$ and Wudang Party School $(106.750 \mathrm{~N}, 26.635 \mathrm{E})$ were most affected by landslides in Wudang.

The main reason is that these schools are either built on steep slopes or too close to steep slopes. Shunhai Primary School is built on an earthy steep slope in Shunhai. The roads pass through the school gate and behind the school, causing the high risk of landslides in the region. Xintian Driving School and Quanlin Driving School are located at the front edge of soil slope, with high risk of landslide. Shuijinhuadu Kindergarten is also located at the front edge of the soil slope. In contrast, the trailing edge of the Wudang Party School is a stone slope, which is easily affected by the collapse. Landslide significantly affects the Xintian First Primary School and Guizhou Normal College in Wudang. For Xintian First Primary School, the risk of landslide comes from the mountain behind the teaching building. For Guizhou Normal College, the risk is from the mountain behind Jianhu Lake. Two small landslides have occurred in this region. Although with the low overall risk of landslide in Guizhou Normal College, the region near the mountain has high risk and probability of landslides.

Urban night lighting area map of Wudang is obtained by extracting the positive region from DMSP/OLS night lighting data of NASA in 2013 (Fig. 3). The lighting area is distributed in the part of city expansion in Guiyang, covering the area of nearly $200 \mathrm{~km}^{2}$. Nearly the half landslide risk regions in Wudang are distributed in the urban regions with high population density. With the highest landslide risk, Xintian and Gaoxin Community Service Centers have the population densities of 1,509 and 2,705 per $\mathrm{km}^{2}$, respectively. High vulnerability of landslides is caused by high population and economic density in urban regions.

Based on the detailed vector boundary file of administrative division, we analyzed the landslide space prediction to collect the landslide prediction data of each township in Wudang. Wherein, Xintianzhai has the highest risk of landslide, with the higher risk and higher risk regions accounting for 5.3 and $21.3 \%$ of total area, respectively. Xiaba Town has the second highest risk of landslide, with the higher risk and high risk regions accounting for 1.3 and $17.9 \%$, respectively. Dongfeng Town has the high risk of landslide, with the high risk and high risk regions accounting for 0.3 and $6.5 \%$, respectively. Shuitian Town has the significant risk of landslide, with the high risk and high risk regions accounting for 0.13 and $7.1 \%$, respectively. In Xinchang and Yangchang Town, the high risk regions are less than $0.1 \%$; the high risk areas are 2.6 and $2.3 \%$, respectively. In Baiyi, Xinpu and Pianpo Town, the high risk regions 
approximate to zero; the high risk regions are $3.2,1.5$ and $1.1 \%$, respectively.

\section{Discussion}

Taking Wudang, Guiyang of Guizhou Province as an example, the work conducted the space prediction of landslide disasters in Karst region by remote sensing, GIS and information-based landslide prediction method. The principal component quantitative method was used to select the relative height difference, slope direction, gradient, type, TWI and LUCC. After extracting the LUCC by SVM-based computer classification and visual interpretation method, we obtained other landslide factors from the DEM based on ArcGIS. The information content of single-factor landslide was calculated by the spatial analysis function of ArcGIS. Then, the information contents of different single-factor layers were summed to obtain the comprehensive information content. Based on the group-occurring landslides and Nature Break method, the landslide information content was divided into high, higher, medium, lower and low risk regions. Wherein, the higher, high and medium risk regions had 42, 56 and 46 potential landslide groups, respectively.

Finally, the landslide space prediction was restricted by the data accuracy and landslide sample data. The former was obtained by the high-resolution remote sensing images and DEM, and the latter should strengthen the landslide monitoring to provide the detailed landslide samples. In addition, the landslide interpretation was conducted with multi-temporal remote sensing images. Due to the contradiction between people and land in the research area, the typicality of most landslides is destroyed after disasters. Therefore, few landslides can be interpreted by the remote sensing image of a phase, which increases the cost of remote sensing interpretation.

\section{Acknowledgement}

The work was supported by Land and Resources Bureau, Wudang, Guiyang. Additionally, this research was funded by the project of the National Science Fuoundation of China(No. 41861035), the project of Guizhou Province Education Department for Tip-top Talent of Science and Technology(Qianjiaohe KY [2016] 082, Qianjiaohe KY [2016] 084 and Qianjiaohe KY[2016]223), and the project of Guizhou Province Science and Tecnology Agency(Qiankehezhicheng [2018] 2776, Qiankehejichu [2016] 1112, Qiankehejichu [2018] 1120). Finally, we thank those anonymous reversers for their constructive suggestions that greatly improved these works.

\section{References}

1. Bălteanu Dan, Chendeş Viorel, Sima Mihaela.,et al.A country-wide spatial assessment of landslide susceptibility in Romania[J]. Geomorphology, 124 (3-4):102-112(2010).

2. Chun-Hung Wu,Su-Chin Chen. Determining landslide susceptibility in Central Taiwan from rainfall and six site factors using the analytical hierarchy process method[J].Geomorphology,112(3-4):190-204(2009).

3. Pradhan B, Lee S. Landslide susceptibility assessment and factor effect analysis: backpropagation artificial neural networks and their comparison with frequency ratio and bivariate logistic regression modelling[J]. Environmental Modelling \& Software, 25(6):747-759(2010).

4. Bonaventura Firman Dwi Wahono.Applications of Statistical and Heuristic Methods for Landslide Susceptibility Assessments[D]. Thesis of Gadjah Mada University(2010).

5. Nandi A, Shakoor A. A GIS-based landslide susceptibility evaluation using bivariate and multivariate statistical analyses[J]. Engineering Geology, 110(1-2):11-20(2010).

6. Pradhan B. Remote sensing and GIS-based landslide hazard analysis and cross-validation using multivariate logistic regression model on three test areas in Malaysia[J]. Advances in Space Research, 45(10): 1244-1256(2010).

7. Isik Y.Landslide susceptibility mapping using frequency ratio, logistic regression, artificial neural networks and their comparison: A case study from Kat landslide(Tokat-Turkey)[J].Computers \& Geosciences,35(6):1125-1138(2009).

8. Kawabata D, Bandibas J. Landslide susceptibility mapping using geological data, a DEM from ASTER images and an Artificial Neural Network (ANN)[J]. Geomorphology, 113(1-2):97-109(2009).

9. Saro Lee, Moung-Jin Lee.Detecting landslide location using KOMPSAT 1 and its application to landslidesusceptibility mapping at the Gangneung area, Korea[J]. Advances in Space Research,38(10):22612271(2006).

10. Dahal R K, Hasegawa S, Nonomura A, et al. GISbased weights-of-evidence modelling of rainfallinduced landslides in small catchments for landslide susceptibility mapping[J]. Environmental Geology, 54(2):311-324(2008).

11. Dahal R K, Hasegawa S, Nonomura A, et al. Predictive modelling of rainfall-induced landslide hazard in the Lesser Himalaya of Nepal based on weights-of-evidence[J]. Geomorphology, 102(34):0-510(2008).

12. Dai F, Lee C. Frequency-volume relation and prediction of rainfall-induced landslides[J]. Engineering Geology, 59(3):253-266(2001).

13. Sengupta A, Gupta S, Anbarasu K. Rainfall thresholds for the initiation of landslide at Lanta Khola in north Sikkim, India[J]. Natural Hazards, 52(1):31-42(2010).

14. Floris M, Bozzano F. Evaluation of landslide reactivation: A modified rainfall threshold model 
based on historical records of rainfall and landslides[J]. Geomorphology, 94(1-2):0-57(2008).

15. Yan Tongzhen, Wu Faquan, Yin Kunlong. Static and Dynamic Regularity of Landslides and Space-time Prognosis of Slope Instability[J]. Earth Science(Journal of China University of Geosciences) ,2:117-133(1989). 